\title{
Assessment of adequate quality and collocation of reference measurements with space-borne hyperspectral infrared instruments to validate retrievals of temperature and water vapour
}

\author{
X. Calbet \\ EUMETSAT, Eumetsat Allee 1, 64295 Darmstadt, Germany \\ Correspondence to: X. Calbet (calbet@eumetsat.int) or (xcalbeta@aemet.es) \\ Received: 10 March 2015 - Published in Atmos. Meas. Tech. Discuss.: 5 June 2015 \\ Revised: 16 November 2015 - Accepted: 24 November 2015 - Published: 15 January 2016
}

\begin{abstract}
A method is presented to assess whether a given reference ground-based point observation, typically a radiosonde measurement, is adequately collocated and sufficiently representative of space-borne hyperspectral infrared instrument measurements. Once this assessment is made, the ground-based data can be used to validate and potentially calibrate, with a high degree of accuracy, the hyperspectral retrievals of temperature and water vapour.
\end{abstract}

\section{Introduction}

Space-borne infrared hyperspectral instruments typically measure Earth views in a spectral range from 600 to $3000 \mathrm{~cm}^{-1}$ wavenumbers with a spectral sampling of about $0.25 \mathrm{~cm}^{-1}$ providing thousands of channels across their full spectral range. From these measurements it is possible to retrieve atmospheric profiles of temperature and water vapour with a relatively high vertical resolution and high degree of accuracy. These (so-called) retrievals can have a temperature accuracy of about $1 \mathrm{~K}$ in layers $1 \mathrm{~km}$ thick and humidity accuracy from 10 to $20 \%$ in layers $2 \mathrm{~km}$ thick within the troposphere (Smith et al., 2001). The algorithms to obtain these retrievals are usually of the following two kinds.

- Regression methods - These are methods based on regression techniques like artificial neural networks, kernel ridge regression or, more simply, a linear regression (see for example Camps-Valls et al., 2012). These methods are usually trained with a representative sample of atmospheric profiles and their corresponding radiances. This training sample can be obtained either by using di- rect measurements of both radiances and atmospheric profiles or by simulating the radiances from the atmospheric profiles using a radiative transfer model. Radiative transfer models simulate the propagation of light in the atmosphere by accepting an atmospheric profile as input and providing radiances as output. The regression methods are later used operationally by providing the measured radiances as input and obtaining the atmospheric profiles as output via the regression.

- Minimization methods - The second kind of retrieval algorithms need a radiative transfer model to operate. In these algorithms, the radiances obtained from the radiative transfer model are matched to the measured ones by modifying the input atmospheric profiles via a minimization algorithm until both calculated and measured radiances coincide within a given error. A well known method in this category is "optimal estimation" (OE Rodgers , 2000).

It is not straight forward to validate these retrievals against independent reference measurements, like for example sondes. Common practice, see for example Tobin et al. (2006), is to calculate the best estimate of the atmospheric profiles from the in situ measurements, therefore minimizing collocation errors, to then directly compare them with the retrievals. Another possibility, when only one sonde measurement is available, is to directly compare the sonde measurement with the retrievals. But, in doing so, important effects which plague these validation exercises can be ignored. Generally, the two most important obstacles that are met when performing these kind of validations are the errors involved in the measurements of the reference profiles and collocation uncertainties 
that might remain between the ground-based reference measurement and the satellite one. Other sources of uncertainties can be an incorrect modelling of the radiative transfer or an unexpected behaviour in the noise characteristics of the hyperspectral instrument.

To effectively take a reference measurement, the error of a particular profile has to be much smaller than the error of its corresponding hyperspectral retrieval. This condition is usually met when hyperspectral retrievals are compared to sondes, which typically have an error of $0.1 \mathrm{~K}$ for temperature and at most $3 \%$ for relative humidity in the lower and mid troposphere (Paukkunen et al., 2001; Miloshevich et al., 2006). It is also necessary that the reference measurements are free of bias and have no systematic errors, a circumstance that is not always met when measuring humidity with certain type of sondes which can have up to a $50 \%$ systematic error in the upper troposphere (e.g., Vömel et al., 2007). This effect could render the comparison ineffective.

An added complication is that the reference measurement usually measures a collection of parcels in the atmosphere which are not exactly the same as the ones measured by the hyperspectral instrument. A radiosonde, for example, measures at one small region or point in the atmosphere and it drifts from the launch location, measuring in different locations and at different times, whereas a hyperspectral instrument measures nearly instantly a large region of the atmosphere with typical footprints of tens of kilometres. These effects contribute to a significant difference between both measurements; this amounts to what is called collocation uncertainty. A notable example is water vapour, which has a high variability in the atmosphere with very small temporal and spatial scales (Vogelmann et al., 2015), making the collocation particularly difficult. In order for the validation to be effective, the collocation uncertainty needs to be much smaller than the error of its corresponding retrieval (Sussmann et al., 2009; Vogelmann et al., 2011).

There are currently two possible strategies to overcome these problems. One of them is to estimate all the errors involved in the validation process, from reference measurement errors to collocation uncertainties plus any other error that could affect the comparison. One such attempt has been done by Pougatchev et al. (2009). Another strategy is to assess whether the global measurement, collocation and radiative transfer modelling errors are small enough to make the validation useful. This is the objective of this paper, where a method to assess the adequacy of an individual reference measurement to a particular retrieval methodology is presented. Since the method, as will be seen below, is based on comparing the satellite measured radiances with the calculated ones using the radiative transfer model and the reference atmospheric profiles, it only applies to retrieval methods based on a radiative transfer model and it is not directly applicable to other retrieval methods (i.e. regression methods trained with measured data).
To illustrate the method, one spectrum from a single Infrared Atmospheric Sounding Interferometer (IASI) field of view is used and four different IASI collocated potential reference profiles are analysed. These data are described in Sect. 2. The method is described in Sect. 3. Finally, a discussion of the method is portrayed in the conclusions.

\section{Data}

\subsection{Raw data}

Infrared hyperspectral data are obtained from the IASI instrument on board the polar orbiting satellite Metop-A. IASI is measuring within the whole spectral range from 645 to $2760 \mathrm{~cm}^{-1}$ with a spectral sampling of $0.25 \mathrm{~cm}^{-1}$, an apodized effective resolution of $0.25 \mathrm{~cm}^{-1}$ and with a spatial resolution of about $12 \mathrm{~km}$ at nadir. One single IASI field of view is analyzed in this study over the Sodankylä observatory, northern Finland (location: $67.368^{\circ} \mathrm{N}, 26.633^{\circ} \mathrm{E}$, 179 ma.s.l.) overpassing the observatory on 17 July 2007 at 08:18 Z. This particular field of view is selected because it is cloud free, making the radiative transfer model calculations simpler. It also has a significant set of accompanying ground-based measurements from the EPS/Metop Sodankylä campaign.

Radiosonde data are from the EPS/Metop Sodankylä campaign, which took place during the time period 4 June to 5 September 2007 (for more details see Calbet et al., 2011). Also, ECMWF analyses have been used either on its own or to complement the radiosonde data. The particular reference temperature and water vapour profiles, which are plotted in Fig. 1, are obtained from the following sources.

- Nearest geo-located ECMWF analysis at 06 Z, which is about 2:30 h before satellite overpass time - This profile will be referred to as "ECMWF".

- Interpolated sonde data from two sonde measurements - A Cryogenic Frost Point Hygrometer (CFH) one, in which the sonde is launched $1 \mathrm{~h}$ before satellite overpass time, and an "in situ" bias corrected RS92 one, in which the sonde is launched $5 \mathrm{~min}$ before satellite overpass time. The interpolation is done in the time domain following Tobin et al. (2006). The "in situ" bias correction is derived from the comparison of the $\mathrm{CFH}$ sonde data with the data from yet another RS92 sonde. These latter two sondes are flown on the same balloon launched $1 \mathrm{~h}$ before satellite overpass time. This profile will be referred to as "Interpolated". In this paper, it is taken as the best estimate of the atmosphere for this hyperspectral observation. See Calbet et al. (2011) for more details.

- The same RS92 sonde launched 5 min before overpass time as the one used to evaluate the "Interpolated" profile, but this time with the humidity being bias corrected 


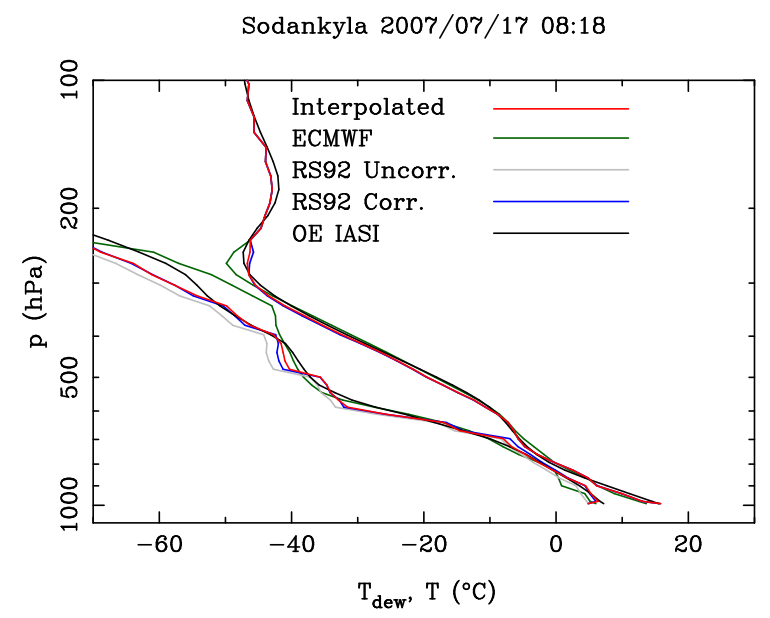

Figure 1. Temperature and dew point temperature of the different profiles used in this paper. The OE IASI retrieval is also shown (in black) for reference purposes only.

following Vömel et al. (2007) and without any kind of interpolation, i.e., using solely data from this RS92 sonde - This data will be referred to as "RS92 Corr.".

- RS92 sonde launched 5 min before overpass time without any kind of bias corrections - This data will be referred to as "RS92 Uncorr.".

It is now worth looking at the different profiles in Fig. 1. They are generally very similar and consistent except for a few differences. The water vapour concentration for "ECMWF" is clearly much higher than the other ones in the upper troposphere/low stratosphere. The "RS92 Uncorr." profile is much drier than the others from mid troposphere up. These differences will show up in the observed minus calculated radiances analysis made below (Figs. 4 and 5).

\subsection{IASI retrievals}

One IASI retrieval is obtained for comparison purposes. The retrieval also constitutes a good starting point to estimate the OE retrieval error, which is essential for the method presented here, but the error could also be calculated from any other realistic atmospheric profile which matches the situation. The IASI retrieval has been calculated following the techniques described in Calbet et al. (2006) and the fine tuning of Calbet (2012). The general description and some particular enhancements and modifications introduced with respect to Calbet et al. (2006) are briefly summarized below:

- Retrievals were obtained using optimal estimation (OE) Rodgers (2000) with physical constraints by prohibiting supersaturation and superadiabaticity.

- All IASI channels from band 1 and 2 have been used, but excluding the ozone band.
- The background state and matrix used in the OE have been obtained from the Chevallier (2002) data set.

- Fine tuning of the OE has been done with collocated ECMWF analyses Calbet (2012), both with respect to bias corrections and measurement error covariance matrix. Due to the significant inaccuracy of ECMWF water vapour analyses (e.g. quite noticeable in Fig. 1), the resulting measurement error covariance matrix used in $\mathrm{OE}$ is clearly overestimated in the water vapour band. This leads to a relatively big expected error in the water vapour retrievals (Fig. 8).

- First guess with which the OE is initialized is the "Interpolated" profile, which is considered to be the best estimate of the atmosphere for this case.

- Radiative transfer model is the optimal spectral sampling (OSS) from Moncet et al. (2008) trained with the Line-By-Line Radiative Transfer Model (LBLRTM) version 11.3.

For illustration purposes the differences of these four profiles against the $\mathrm{OE}$ retrieval are plotted in Fig. 2. It can be seen that the differences depend very strongly on the reference profile used. While most profiles do not deviate significantly from the OE retrieval, the "ECMWF" profile does show comparatively large differences. It is worth noting that all the radiosonde data come from the EPS/Metop Sodankylä campaign and therefore has not been assimilated into any Numerical Weather Prediction (NWP) model.

\section{Method}

The assessment method consists of two steps. In the first one the observed radiances are compared to the calculated ones. The second step consists in converting the mentioned radiance differences into atmospheric state differences.

\subsection{Observed minus calculated radiances}

To get a sense of how well the reference atmospheric profiles are representative of the atmosphere at the IASI field of view, the IASI measured radiances can be compared to the calculated ones using a radiative transfer model. This effectively means that the measured atmospheric profile, the radiative transfer model and the IASI radiances are consistent among themselves within their measurement errors.

The calculated radiances are obtained by applying a radiative transfer model to the measured reference atmospheric profile and its corresponding surface properties. It is important to note here that the atmospheric and surface parameters should come, as much as possible, from measurements or any other sources that are independent from the IASI measurements. In other words, the atmospheric profile and surface properties should ideally not be derived from the IASI 

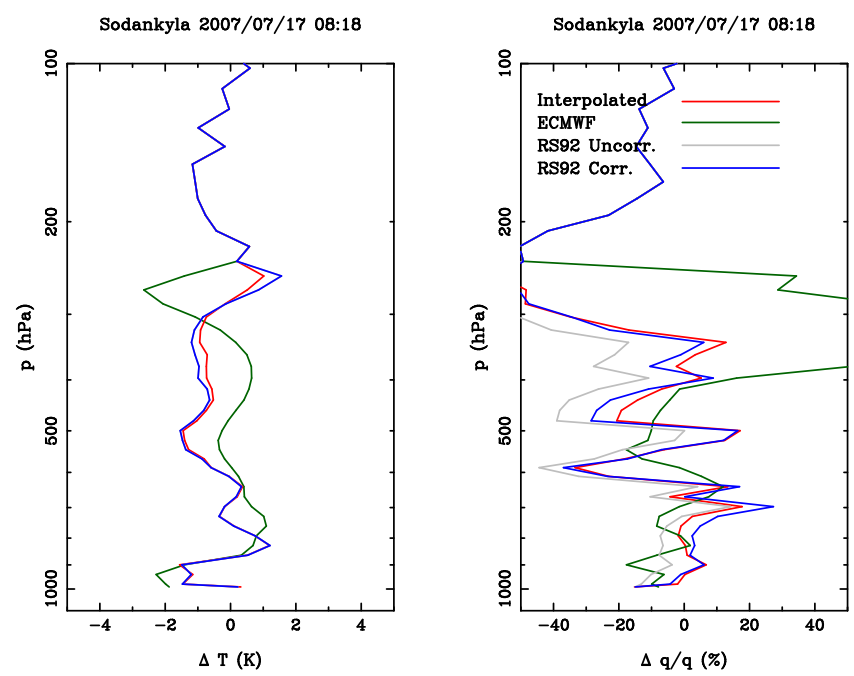

Figure 2. Difference of the reference profiles minus the OE retrieval.

measurements, like they would be if a retrieval is performed or some other similar kind of technique is used. The reason behind this is that the final goal of the study is to make an assessment of the reference profile and not of the retrieval. Using data obtained from IASI radiances would artificially increase the agreement between the calculated and measured radiances, thus affecting our assessment method. In the most extreme case, when using atmospheric profiles and quantities that are all derived or retrieved from IASI radiances, it is the retrieval that is assessed and not the reference profiles. It is not always possible to meet this requirement in practice, and it is often the case that some of the parameters needed as input for the radiative transfer model are missing, as typically happens with surface emissivity or surface skin temperature. If this is the case, the number of retrieved parameters should be minimized as much as possible.

In particular, in this paper the calculated radiances are obtained using the following methods.

- The temperature and water vapour profiles are used based on radiosonde measurements ("Interpolated", "RS92 Corr." and "RS92 Uncorr."), which are complemented in the upper layers, where the sonde instruments reach their limit, with the "ECMWF" profile. See Calbet et al. (2011) for more details.

- The ozone profile is obtained from the ECMWF analysis for all cases.

- The radiative transfer model used is OSS Moncet et al. (2008), trained with LBLRTM 11.3.

- Surface emissivity is the one corresponding to old pine leaf from the MODIS UCSB emissivity library MODIS Emissivity (1999). This surface emissivity seems to be

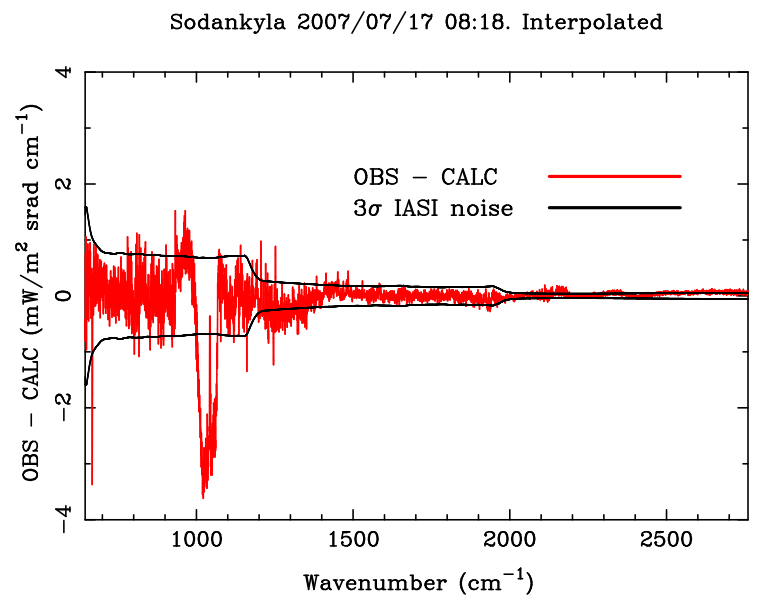

Figure 3. IASI observed minus calculated radiances (OBS-CALC) for the "Interpolated" profile.

the most appropriate for this site, which is covered by an old pine forest.

- Surface skin temperature measurements are not available and had to be retrieved from the spectra by matching the calculated radiances to the observed ones.

The difference of the observed minus the calculated radiances are shown in Figs. 3, 4, 5 and 6. The $3 \sigma$ IASI noise is plotted in these figures as a black line. Some features are worth noting. The observed minus calculated radiances do not fit well in the ozone band $\left(\sim 1000 \mathrm{~cm}^{-1}\right)$, indicating that most likely the ozone profile (obtained from ECMWF in all cases) is not very accurate. Radiance differences do not match in IASI band $3\left(\sim 2000 \mathrm{~cm}^{-1}\right.$ and above), which is caused by inadequate modelling of the part of the spectrum that is affected by solar radiation. The "Interpolated" and "RS92 Corr." profiles (Figs. 3 and 6) fit very well along the rest of the spectrum and mostly lie within the $3 \sigma$ IASI noise lines. The "ECMWF" profile calculated radiances do not match the IASI observed ones very well (Fig. 4), especially in the water vapour band $\left(1400\right.$ to $1900 \mathrm{~cm}^{-1}$ ), caused by the positive deviation in the upper troposphere of the ECMWF water vapour profile as evidenced in Fig. 1. The "RS92 Uncorr." profile does not match well in the water vapour band either (Fig. 5), showing an opposite sign in the radiance differences with respect to ECMWF, caused by the drier water vapour profile in the upper layers (Fig. 1).

From these four observed minus calculated radiance figures (Figs. 3, 4, 5 and 6), it can be concluded that the radiative transfer calculations applied to two of the temperature and humidity profiles, "Interpolated" and "RS92 Corr.", are consistent with IASI measurements, and the other two, "ECMWF" and "RS92 Uncorr.", are not. Therefore the former two profiles are suited for validation or calibration of IASI retrievals and the latter two are not. The question that immediately follows is whether an objective criteria can 


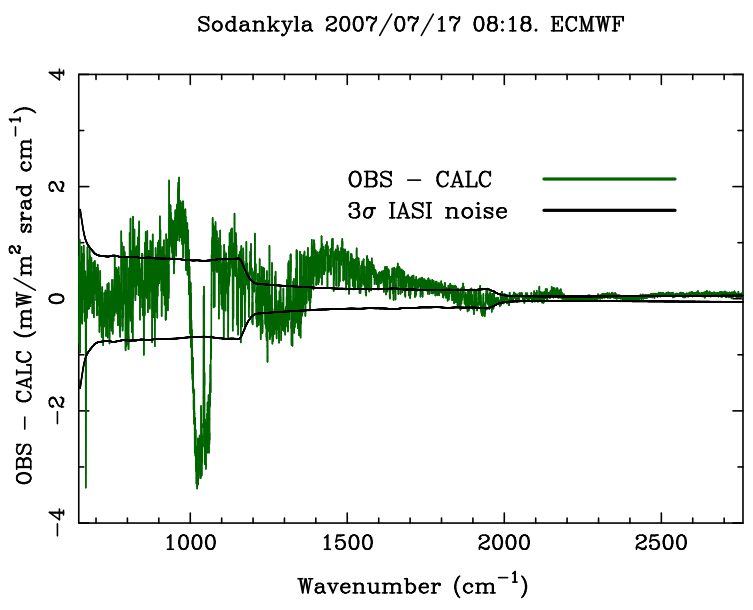

Figure 4. IASI observed minus calculated radiances (OBS-CALC) for the "ECMWF" profile.

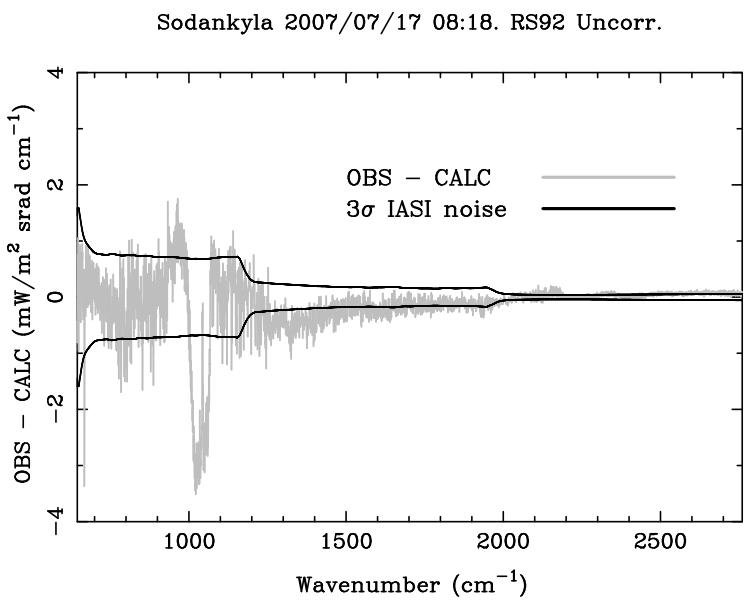

Figure 5. IASI observed minus calculated radiances (OBS-CALC) for the "RS92 Uncorr." profile.

be established to select or reject particular reference atmospheric profiles. This will be developed in the following section.

\subsection{Atmospheric profile errors}

The natural quantity to set up as a threshold to which the different reference atmospheric profile errors can be compared to is the retrieval error, which arises directly from the OE theory of Rodgers (2000). If the atmospheric profile errors are much larger than the errors achieved by OE, then the profiles are not suited as reference measurements. If, on the other hand, the atmospheric profile errors are smaller or of the order of the OE retrieval errors, then these profiles can be used as reference measurements. Consequently, the question at this stage is how to convert the observed minus calculated radiance errors into profile errors in the atmospheric state space.

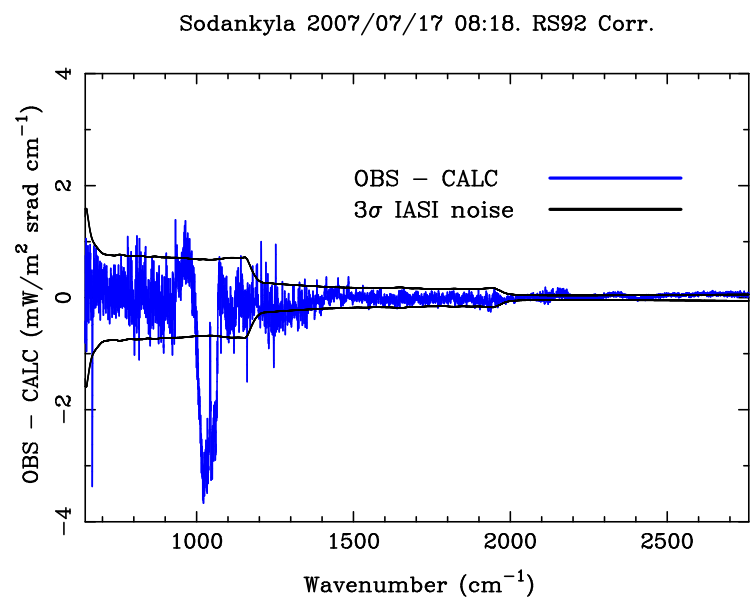

Figure 6. IASI observed minus calculated radiances (OBS-CALC) for the "RS92 Corr." profile.

The directly observed minus calculated radiances for one particular IASI field of view (as in Figs. 3, 4, 5 and 6) constitute individual samples of these differences. To estimate the actual errors of these differences it is necessary to estimate their covariances by calculating the standard deviation within a big enough sample. To accomplish this, the values from neighbouring channels are used. This is done by obtaining the square root of the moving average over a spectrum of the square of the observed minus calculated radiances. The length of the window of the moving average which is found to be useful in practice is 500 channels. In doing so, it is implicitly assumed that the statistical probability distribution of the errors of the 500 neighbouring channels are similar. In general this will most likely be the case, but in some circumstances, like particular spectral absorption lines, might not be completely accurate.

The estimation of these standard deviations of the radiances are shown in Fig. 7 for all four cases. The ozone band is not plotted in this figure because of the big uncertainty shown in this region due to a not well characterized ozone profile. Note the very low standard deviation, below $1 \sigma$ IASI instrument noise, for some regions of the spectrum for the "Interpolated" and "RS92 Corr." profiles, as already acknowledged in Calbet et al. (2011). Also recall that there is only one parameter retrieved from IASI radiances when obtaining the calculated radiances, which is the surface skin temperature.

The standard deviation of the radiances difference (Fig. 7) needs to be translated from radiance space into atmospheric profile space. To do this, the OE theory Rodgers (2000) needs to be recalled by expressing the cost function, $J$, as

$$
\begin{array}{r}
J=(y-F(x))^{\mathrm{T}} \mathbf{S}_{\epsilon}^{-1}(y-F(x)) \\
+\left(x-x_{a}\right)^{\mathrm{T}} \mathbf{S}_{a}^{-1}\left(x-x_{a}\right),
\end{array}
$$

where $\boldsymbol{y}$ is the hyperspectral measurement, $\boldsymbol{F}$ is the radiative transfer model, $\mathbf{S}_{\epsilon}$ is the measurement error covariance matrix used in the IASI retrievals, $\boldsymbol{x}$ is the atmospheric profile 


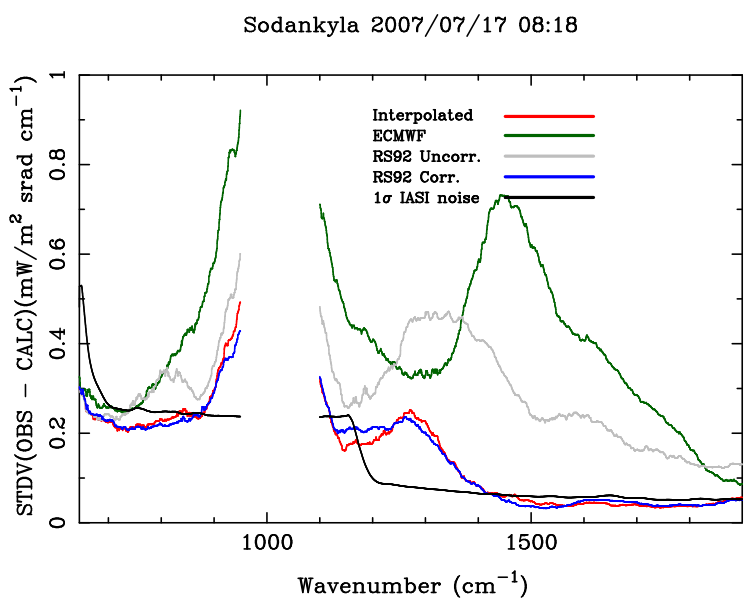

Figure 7. Estimation of the standard deviation of the observed minus calculated radiance differences for each reference atmospheric profile, having removed the ozone band.

state, $x_{\mathrm{a}}$ is the background state and $\mathbf{S}_{\mathrm{a}}$ is the background covariance matrix. This cost function is usually linearised around an atmospheric state close to the final solution, $x_{x}$,

$$
\begin{array}{r}
J \approx(\delta y-\mathbf{K} \delta x)^{\mathrm{T}} \mathbf{S}_{\epsilon}^{-1}(\delta y-\mathbf{K} \delta x) \\
+\left(\delta x-\delta x_{\mathrm{a}}\right)^{\mathrm{T}} \mathbf{S}_{\mathrm{a}}^{-1}\left(\delta x-\delta x_{\mathrm{a}}\right),
\end{array}
$$

where $\mathbf{K}$ is the Jacobian of $\boldsymbol{F}$ at the linearization point $x_{x}$, $\delta x=x-x_{x}, \delta x_{\mathrm{a}}=x_{\mathrm{a}}-x_{x}$ and $\delta y=y-F\left(x_{x}\right)$. To find the most likely atmospheric state or retrieval, $\hat{x}_{\mathrm{O}}$, corresponding to a particular IASI observation, $y=y_{\mathrm{O}}$, the derivative of $J$ with respect to $\delta x$ is set to zero, giving as a final retrieval solution

$\delta \hat{x}_{\mathrm{O}}=\left(\mathbf{K}^{\mathrm{T}} \mathbf{S}_{\epsilon}^{-1} \mathbf{K}+\mathbf{S}_{\mathrm{a}}^{-1}\right)^{-1}\left(\mathbf{K}^{\mathrm{T}} \mathbf{S}_{\epsilon}^{-1} \delta y_{\mathrm{O}}+\mathbf{S}_{\mathrm{a}}^{-1} \delta x_{\mathrm{a}}\right)$,

where $\delta \hat{x}_{\mathrm{O}}=\hat{x}_{\mathrm{O}}-x_{x}$ and $\delta y_{\mathrm{O}}=y_{\mathrm{O}}-F\left(x_{x}\right)$. It is known that the error or covariance of this retrieval solution Rodgers (2000) is

$\mathbf{S}_{x}=\left(\mathbf{K}^{\mathrm{T}} \mathbf{S}_{\epsilon}^{-1} \mathbf{K}+\mathbf{S}_{\mathrm{a}}^{-1}\right)^{-1}$,

which is a quantity that will be needed later. A similar technique can be applied to obtain the most likely state vector, $x_{\mathrm{C}}$, corresponding to the calculated radiance, $y_{\mathrm{C}}$, obtained from applying a radiative transfer model to any of the reference atmospheric profiles,

$\delta \hat{x}_{\mathrm{C}}=\left(\mathbf{K}^{\mathrm{T}} \mathbf{S}_{\epsilon}^{-1} \mathbf{K}+\mathbf{S}_{\mathrm{a}}^{-1}\right)^{-1}\left(\mathbf{K}^{\mathrm{T}} \mathbf{S}_{\epsilon}^{-1} \delta y_{\mathrm{C}}+\mathbf{S}_{\mathrm{a}}^{-1} \delta x_{\mathrm{a}}\right)$,

where $\delta \hat{x}_{\mathrm{C}}=\hat{x}_{\mathrm{C}}-x_{x}$ and $\delta y_{\mathrm{C}}=y_{\mathrm{C}}-F\left(x_{x}\right)$. The difference between the two retrieved state vectors, $\Delta \hat{x}=\hat{x}_{\mathrm{O}}-\hat{x}_{\mathrm{C}}$, gives a quantity that measures the error in the state vector when using the calculated radiances, $y_{\mathrm{C}}$, instead of the observed ones, $y_{\mathrm{O}}$. In other words, $\Delta \hat{x}$ provides a measure of the reference state quality and collocation error plus any errors we might have done in the radiative transfer model assumptions. Solving for $\Delta \hat{x}$ gives

$\Delta \hat{x}=\left(\mathbf{K}^{\mathrm{T}} \mathbf{S}_{\epsilon}^{-1} \mathbf{K}+\mathbf{S}_{\mathrm{a}}^{-1}\right)^{-1}\left(\mathbf{K}^{\mathrm{T}} \mathbf{S}_{\epsilon}^{-1} \Delta y\right)$,
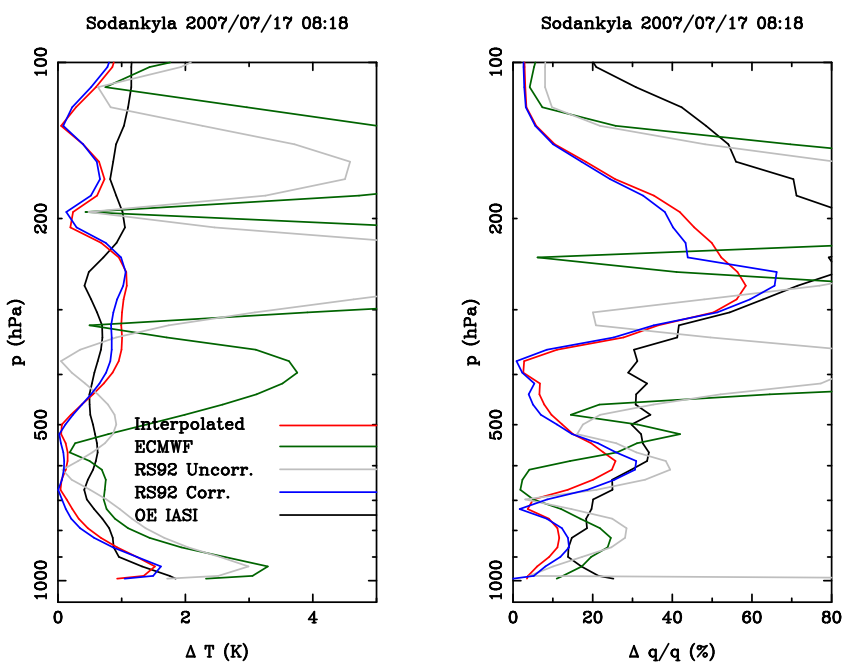

Figure 8. Retrieval error (diagonal of Eq. (4) in black) and collocation and adequacy errors ( $\Delta \hat{x}$ from Eq. 6$)$ for the different reference profiles.

where $\Delta y=y_{\mathrm{O}}-y_{c}$. This last equation permits the conversion of the standard deviation radiance difference, $\Delta y$, into atmospheric state space, $\Delta \hat{x}$. The latter will be referred to as collocation and adequacy errors of the reference profiles.

Having all the necessary elements, it is now possible to define a criteria to evaluate whether a given atmospheric profile measurement effectively constitutes a reference profile for IASI. A given atmospheric profile measurement can be classified as a useful reference for IASI if the collocation and adequacy errors in the atmospheric profiles, $\Delta \hat{x}$ from Eq. (6), is below or of the order of the retrieval error, $\mathbf{S}_{x}$ from Eq. (4). The results for the four profiles are shown in Fig. 8 for temperature and water vapour, along with the estimated IASI retrieval error (in black) for comparison. It can be verified that the collocation and adequacy errors of the "Interpolated" and "RS92 Corr." atmospheric profiles are of the same order of magnitude as the IASI retrieval error. Therefore, these two cases would qualify as reference measurements for the retrievals. The remaining two profiles, "ECMWF" and the "RS92 Uncorr." show collocation and adequacy errors that are much larger than the retrieval errors and should not be used for validation or calibration purposes.

\section{Conclusions}

The conventional methodology to validate, and possibly calibrate, infrared hyperspectral sounding retrievals with reference measurements (e.g. sondes) or other kind (e.g. NWP fields) of atmospheric profiles of temperature and water vapour is to first collocate the reference profiles with the hyperspectral instrument fields of view. Later, a comparison of the reference profiles and the hyperspectral retrievals is made to finally obtain some kind of parameter which gives the de- 
gree of coincidence between both, typically bias and standard deviation statistics. Issues like collocation uncertainties, systematic errors in the humidity measurements, etc. can easily be introduced in the comparison exercise. As a consequence and as it has been shown in this paper, this methodology would, in general, grossly overestimate the uncertainties of the hyperspectral retrievals.

In this paper we propose the introduction of an additional step, after the collocation is performed, to the common validation methodology which consists in assessing the proper collocation and quality of the reference profiles with respect to the hyperspectral retrievals. The way to perform this assessment, in summary, consists of first obtaining the calculated radiances by using the reference profile with as few retrieved parameters from hyperspectral radiances as possible. These calculated radiances are then compared to the ones observed by the hyperspectral instrument, and a standard deviation as a function of wavenumber is obtained for the whole spectrum and for each particular field of view. This radiance standard deviation is then translated into an error in the atmospheric state space via Eq. (6), which will englobe the overall errors in collocation and adequacy of the measurements with respect to the hyperspectral instrument. These kind of errors could be accuracy of the reference measurement profile, collocation uncertainties, errors in the radiative transfer modelling, non-nominal noise behaviour of the hyperspectral instrument, etc. If these collocation and adequacy errors are much bigger than the expected retrieval errors then these particular profiles should not be used for validation. Otherwise, the atmospheric profiles do constitute a reference measurement which can be used for validation and possibly calibration of the hyperspectral retrievals. In other words, this assessment checks whether the measured atmospheric profiles along with the used radiative transfer modelling and the hyperspectral instrument measurements are consistent among each other. Another way to look at this problem is to understand that if the observed and calculated radiances are not consistent and compatible with each other, it will be very difficult, if not impossible, to obtain retrievals that match, within the uncertainty bounds, the measured atmospheric reference profiles.

As an illustration of the method, four potential reference profiles have been tested against one particular IASI field of view measurement. Results are shown in Fig. 8. In these particular cases, the "Interpolated" (an interpolation of CFH launched $1 \mathrm{~h}$ before satellite overpass time and "in situ" humidity bias corrected RS92 sonde launched $5 \mathrm{~min}$ before satellite overpass time) and "RS92 Corr." (Vömel et al., 2007 humidity bias corrected RS92 sonde launched $5 \mathrm{~min}$ before satellite overpass time) profiles do meet the criteria and can be used as reference atmospheric profiles. The other two, the "ECMWF" (ECMWF analysis) and the "RS92 Uncorr." (uncorrected RS92 sonde launched 5 min before overpass time) profiles do not qualify as proper reference calibration or validation profiles. A feeling of what impact in selecting one type of reference profile over another in the validation of the OE retrievals can be seen in Fig. 2. The comparison with the valid profiles that meet the selection criteria would clearly provide a better result than the comparison with the rejected ones.

An added benefit to this technique is that if there any significant issues with the comparison of profiles and retrievals they will show up in this adequacy assessment. Possible sources of errors that have been identified are large biases in the humidity measurements of RS92 radiosonde sensors Calbet et al. (2011) and possibly water vapour continuum deficiencies in the radiative transfer model Newman (2012).

The technique shown in this paper is indeed a long process, and some effort needs to be invested in order to understand what are all the issues affecting the reference measurements as compared to infrared hyperspectral observations until a match like the one for the "Interpolated" profiles (Fig. 3) is obtained. It is usually mandatory to understand many of the most important issues affecting all the measurements. Questions like systematic errors in the sonde humidity measurements, cloud contamination of the infrared hyperspectral observations, collocation uncertainty, calculation of the best estimate of the atmosphere, proper radiative transfer modelling, use of proper saturation water vapour function and others need to be well understood. Another downside is that the validation sample size can be reduced greatly if many of the observations are discarded because they do not meet the here described assessment criteria. Also, this method can be applied to species which are frequently measured in the atmosphere, such as temperature and water vapour, but it would be more difficult to apply these techniques to other components which are less often measured, such as atmospheric trace gases. On the positive side, the final selected atmospheric profiles, that have indeed passed the assessment criteria, can then be taken as truly reference profiles to validate infrared hyperspectral retrievals.

Edited by: R. Sussmann

\section{References}

Calbet, X., Schlüssel, P., Hultberg, T., Phillips, P., August, T., Validation of the operational IASI level 2 processor using AIRS and ECMWF data, Adv. Space Res., 37, 2299-2305, 2006.

Calbet, X.: Determination of the best optimal estimation parameters for validation of infrared hyperspectral sounding retrievals, arXiv:1205.3012 [physics], 2012.

Calbet, X., Kivi, R., Tjemkes, S., Montagner, F., and Stuhlmann, R.: Matching radiative transfer models and radiosonde data from the EPS/Metop Sodankylä campaign to IASI measurements, Atmos. Meas. Tech., 4, 1177-1189, doi:10.5194/amt-4-1177-2011, 2011. 
Camps-Valls, G., Munoz-Mari, J., Gomez-Chova, L., Guanter, L., Calbet, X.: Nonlinear Statistical Retrieval of Atmospheric Profiles From MetOp-IASI and MTG-IRS Infrared Sounding Data, IEEE Trans. Geosci. Remote Sens., 50, 1759-1769, 2012.

Chevallier, F.: Sampled databases of 60-level atmospheric profiles from the ECMWF analyses, NWP SAF Doc. No. NWPSAF-ECTR-004, 2002.

MODIS UCSB Emissivity Library: available at: http://www.icess. ucsb.edu/modis/EMIS/html/em.html (last access: 22 December 2015), 1999.

Miloshevich, L. M., Vömel, H., Whiteman, D. N., Lesht, B. M., Schmidlin, F. J., and Russo, F.: Absolute accuracy of water vapor measurements from six operational radiosonde types launched during AWEX-G and implications for AIRS validation, J. Geophys. Res., 111, D09S10, doi:10.1029/2005JD006083, 2006.

Moncet, J. and G. Uymin and A.E. Lipton and H.E. Snell: Infrared radiance modeling by optimal spectral sampling, J. Atmos. Sci., 65, 3917-3934, 2008.

Newman, S. M.: Report on the impact of changes in the water vapour continuum arising from the CAVIAR consortium on satellite remote sensing, Met Office RC13C Key Deliverable Report, February 2012.

Paukkunen, A., Antikainen, V., and Jauhiainen, H.: Accuracy and performance of the new Vaisala RS90 radiosonde in operational use, paper presented at 11th Symposium on Meteorological Observations and Instrumentation, Am. Meteorol. Soc., Albuquerque, N. M., 14-18 January 2001.

Pougatchev, N., August, T., Calbet, X., Hultberg, T., Oduleye, O., Schlüssel, P., Stiller, B., Germain, K. St., and Bingham, G.: IASI temperature and water vapor retrievals - error assessment and validation, Atmos. Chem. Phys., 9, 6453-6458, doi:10.5194/acp9-6453-2009, 2009.

Rodgers, C. D.: Inverse methods for atmospheric sounding, Theory and practice, World Scientific, Singapore, 2000.
Smith, W. L. Sr., Harrison, F., Hinton, D., Miller, J., Bythe, M., Zhou, D., Revercomb, H., Best, F., Huang, H., Knuteson, R., Tobin, D., Velden, C. S., Bingham, G., Huppi, R., Thurgood, A., Zollinger, L., Epslin, R., and Petersen, R.: The Geosynchronous Imaging Fourier Transform Spectrometer (GIFTS) (Invited Presentation), The 11th Conference on Satellite Meteorology and Oceanography (Madison, WI), 2001.

Sussmann, R., Borsdorff, T., Rettinger, M., Camy-Peyret, C., Demoulin, P., Duchatelet, P., Mahieu, E., and Servais, C.: Technical Note: Harmonized retrieval of column-integrated atmospheric water vapor from the FTIR network - first examples for longterm records and station trends, Atmos. Chem. Phys., 9, 89878999, doi:10.5194/acp-9-8987-2009, 2009.

Tobin, D. C., Revercomb, H. E., Knuteson, R. O., Lesht, B. M., Strow, L. L., Hannon, S. E., Feltz, W. F., Moy, L. A., Fetzer, E. J., and Cress, T. S.: Atmospheric radiation measurement site atmospheric state best estimates for atmospheric infrared sounder temperature and water vapor retrieval validation, J. Geophys. Res., 111, D09S14, doi:10.1029/2005JD006103, 2006.

Vogelmann, H., Sussmann, R., Trickl, T., and Borsdorff, T.: Intercomparison of atmospheric water vapor soundings from the differential absorption lidar (DIAL) and the solar FTIR system on Mt. Zugspitze, Atmos. Meas. Tech., 4, 835-841, doi:10.5194/amt-4-835-2011, 2011.

Vogelmann, H., Sussmann, R., Trickl, T., and Reichert, A.: Spatiotemporal variability of water vapor investigated using lidar and FTIR vertical soundings above the Zugspitze, Atmos. Chem. Phys., 15, 3135-3148, doi:10.5194/acp-15-3135-2015, 2015.

Vömel, H., Selkirk, H., Miloshevich, L., Valverde-Canossa, J., Valdés, J., Kyrö, E., Kivi, R., Stolz, W., Peng, G., and Diaz, J. A.: Radiation Dry Bias of the Vaisala RS92 Humidity Sensor, J. Atmos. Oceanic Technol., 24, 953-963, 2007. 